\title{
Interactive comment on "Mid-level convection in a warm conveyor belt accelerates the jet stream" by Nicolas Blanchard et al.
}

\author{
Nicolas Blanchard et al. \\ florian.pantillon@aero.obs-mip.fr \\ Received and published: 11 January 2021
}

We thank the Referee for his/her time and his/her constructive comments. We have complied with most of the proposed changes. In the following, the comments made by the Referee appear in black, while our replies are in blue.

Blanchard et al. present a detailed analysis of convection embedded in a WCB and how this affects the upper-tropospheric flow. The study is based on observations taken during the North Atlantic Waveguide and Downstream Impact Experiment and convection-permitting simulations. A reference simulation (REF) generally agrees with the observations and represents key features such as the WCB outflow, a dry region below this outflow and the cloud head associated with the bent-back warm front. A 
second simulation is performed with latent heating exchanges due to cloud processes being turned off (NODIA). A comparison of the two simulations reveals that elongated bands of negative PV are missing the the NODIA simulation pointing to their diabatic origin. Indeed, the analysis of trajectories and vertical cross section through the WCB suggests that mid-level convection embedded in the WCB is responsible for generating the bands of negative PV in a vertically sheared environment. This is in line with recent studies by Harvey et al. (2020) and Oertel et al. (2020). The study is well written, the figures are mostly clear and the methods are sound. As the paper confirms recent research using novel observations and a slightly different approach (simulations with latent heat release switched on/off), I recommend the article to be published in WCDD after the following comments have been addressed.

\section{Broad comments}

1) The REF and NODIA simulations are compared qualitatively throughout the paper. To my impression it would be helpful if the authors provided quantitative estimates of the differences between the simulations since it is sometimes difficult to spot the differences by eye. As an alternative, difference plots would help the reader to fully appreciate the differences (e.g, Fig. 3, 8) which are discussed in the text.

As also suggested by Referee 1, the Heidke Skill Score is now computed when comparing brightness temperatures simulated by Meso-NH and measured by MSG, quantitative statements are included in the comparison of wind speed between RASTA observations and Meso-NH simulations, while the bias and the root-mean square error are given for the comparison between wind speed, potential temperature and relative humidity measured by the dropsondes and simulated by REF and NODIA.

2) The individual subsections are quite often introduced by describing what is shown in the figures. These descriptions are not necessary since they are also provided in the figure captions. Instead, it would be helpful if the authors described the purpose of each subsection in one to two sentences. This would help to guide the reader through the manuscript.

Interactive

comment

Printer-friendly version

Discussion paper 
As suggested, the subsection headers have been rephrased to introduce their topic rather than the figures they describe.

\section{Minor comments}

I. 2: Please clarify that "their" is referring to WCBs and not to "ridges". Changed to "the representation of WCBs"

Interactive

comment

I. 9: Since the "mesoscale structures" are mentioned here for the first time. Please specify what the "mesoscale structures" are. Are these the tropopause fold and the jet stream core? We removed "mesoscale" as we refer to the "fine-scale observations of cloud and wind structures acquired with airborne Doppler radar and dropsonde"

I. 22: Also PV gradients along zonal flows form a waveguide. Please include this as well. Included

I. 32: l'd suggest to also cite at least one of the early studies, e.g., by Browning et al. (1973) and Harrold (1973). The study of Harrold (1973) is now cited.

I. 32: Other studies state that WCBs are characterized by "rapid ascent" (e.g., Eckardt et al. 2004). Compared to deep convection the WCB ascent may be considered as "slow". Perhaps specify that the ascent is slow compared to deep convective systems. We removed "slowly"

I. 36: Please specify what "This" is referring to. Changed to "This source"

I. 40: Consider to use "Accordingly" instead of "Thus" to avoid the use of the same wording in two consecutive sentences. Changed

I. 52: Please provide a reference for the statement "persisted for several weeks". The reference to Schäfler et al. (2018) has been added.

I. 72: Specify here that RASTA is a cloud radar. Added

I. 87: Is it only the latent heat exchange which is set to zero or are there also other 
diabatic processes set to zero? We added "Note that the other parameterizations (radiation, turbulence, shallow convection) also exchange heat in the atmosphere, but WCDD in a negligible way compared to cloudy processes."

I. 91: Why are you defining three 3-D passive tracers at each grid point and not only one tracer per grid point? We added "Three scalar tracers per grid point allow to follow the three dimensional position of each air parcel."

I. 98: According to e.g. Browning et al. (1986), WCBs start to ascend from the planetary boundary layer. In terms of their terminology: Are you really identifying a WCB as it was originally defined or is it convection that is embedded in a slantwise ascending WCB? We do not claim to formally identify a WCB. As stated, "Selected ascents thus do not perform a full ascent from the boundary layer to the upper troposphere". We clarify that they "may not all belong to actual WCB trajectories" and refer to Blanchard et al. (2020) for a discussion.

I. 106: Please specify that it is 2 October 11:00 UTC. Added

I. 107: I assume you are meaning "in the eastern half" of the simulation domain. "East of the simulation domain" would actually be outside the domain in Fig. 1. Corrected

I. 114: In the region of the cyclonically turning WCB the BT is lower than observed by MSG. In contrast, in NODIA the BTs are similar to the observed values. Do you have any hypothesis why this might be the case? Thanks for pointing this. The underestimation of the BTs was an artifact due to the cloud properties that were used to compute the BTs. It has been corrected.

I. 113-121: It would be very helpful if you labeled some of the key features in Fig. 1 (e.g., cloud head, PV tongue). Added

I. 132: Please consider to indicate the flight direction (e.g., as an arrow) in Fig. 1a. Added

I. 141: To my impression the slope also indicates the location of the cold conveyor belt

Interactive

comment 
which is located below the cloud shield associated with the WCB. If the authors come to the same conclusion this should be mentioned in the text. We agree and mention WCDD this in the text.

I. 147: Consider to replace "until" with "reaching down to". Changed to "reaching"

I. 160: Can the authors comment on whether this low-level jet is also part of the cold conveyor belt? We commented that "The low-level jet likely corresponds to the cold conveyor belt with possible orographic influence."

I. 162: "close to those measured" is a quite qualitative statement. Could you either show a difference plot of the modeled and observed wind speed or provide a quantitative measure such as RMSE? Also showing a scatter plot of observed vs modeled wind speeds could provide a more quantitative estimate of the differences. We prefer to keep focus on the impact of the cloud diabatic impact. However, we added "with a bias of $0.5 \mathrm{~m} \mathrm{~s}^{-1}$ and the root-mean square error of $3.3 \mathrm{~m} \mathrm{~s}^{-1 \text { " }}$ to provide the reader with a quantitative statement.

I. 165: Consider to remind the reader that you have selected all ascents with $w>0.3$ $\mathrm{m} \mathrm{s}^{-1}$. Or are you showing air parcels that fulfill the ascent criterion of $150 \mathrm{hPa}$ in $12 \mathrm{~h}$ ? Please clarify. We added "(that fulfill the ascent criterion of $150 \mathrm{hPa}$ in $12 \mathrm{~h}$ )".

I. 171: Also here, a quantitative statement on the differences would be very helpful. We added "The maximum value is equal to $38 \mathrm{~m} \mathrm{~s}^{-1}$, a value lower than the maximum of $42 \mathrm{~m} \mathrm{~s}^{-1}$ obtained for REF."

I. 177: Write "profiles" instead of "profile". Changed

I. 180-209: When comparing observations to modeled values at individual grid points, differences might occur due to minor spatial shifts between simulations and observation. To account for these spatial displacements, I suggest to consider the values at several neighboring grid points and to show their variability in Fig. 4. E.g. showing the median value of the grid points together with the interquartile range could be one way to

Interactive

comment 
estimate the sampling uncertainty. As one may expect, the simulated fields are rather smooth compared to observations from radiosondes (see curves on Fig. 4). They show zonal gradients (see Fig. 3) but these precisely allow to assess the horizontal extent of the simulated features. For these reasons, and for the sake of visibility, we prefer to show the simulated values at the nearest grid point only. This further allows us to calculate the bias and root-mean square error between the dropsonde measurements and the simulated values for wind speed, potential temperature and relative humidity.

WCDD

I. 215: To my impression there are only two regions of high ascent frequency. One is associated with the bent back warm front and the second region can be found over Greenland. So, what is the reason for splitting the ascent along the bent back warm front in two regions? Please explain in the text. We do not share your impression, because the area north of the cyclone does not overlap with the bent-back front. To illustrate this point, we have added "(as shown in Sec. 3.2)" after "It corresponds to the WCB outflow region overflown by the aircraft".

I. 223: How did you investigate whether the ascents are produced by the warm front dynamics or by orographic forcing? We did not investigate their origin in detail but their presence in NODIA clearly shows it is not diabatic. The sentence has been rephrased and is now "They are likely produced by the combined effect of the warm front dynamics and orographic forcing caused by the Greenland Plateau."

I. 233: I assume it is Fig. 6a. Corrected

I. 234: I assume it is Fig. 6b. Corrected

I. 235: I assume it is Figs. 6a,b. Corrected

I. 236: I assume it is Fig. 6a. Corrected

I. 237: I assume it is Fig. 6b. Corrected

I. 239: Correct to Fig. 6a. Corrected

Interactive comment 
I. 299: Why are you referring to the brown circles? As far as I understand correctly, the red stars in Fig. $6 a$ indicate the position of trajectories closest to the time shown in Fig. 9. Changed to "red stars"

I. 307: The rapid segments are not only found in regions of high $\theta_{e}$, but especially in regions with high $\theta_{e}$ gradients. This should be mentioned in the discussion. Added

Interactive

comment

I. 307 and the following paragraphs: It is not quite clear to me why the focus is on 2 October 2 UTC. The differences between REF and NODIA in terms of upper-tropospheric PV (at $320 \mathrm{~K}$ ) are considerably larger at $06 \mathrm{UTC}$. In fact, at $320 \mathrm{~K}$ differences in PV at 2 UTC are very difficult to identify. It seems that at 2 UTC the negative PV is mostly located in the mid-troposphere. So, could you comment on the processes leading to the negative PV at $320 \mathrm{~K}$ at 06 UTC? Since the differences between REF and NODIA are pronounced at 06 UTC, the negative PV is likely not only a result of isentropic advection. We agree that differences on the 320-K isentropic level are larger at 06 UTC. However, the negative PV bands have already formed and convection has weakened at that time. We clarified the focus on the early hours at the beginning of the Section: "The origin of the negative PV bands is now investigated in the region where both the anticyclonic ascents start (red stars in Fig. 6a) and the elongated negative PV bands found in the WCB outflow region appear to form (box in Fig. 8e). Furthermore, time evolutions have shown that anticyclonic rapid segments are most numerous during the early simulation hours (see black boxplots in Fig. 7a)."

I. 309: Please provide the coordinates of the rapid segments that are located further southwestward. We added "(around $56^{\circ} \mathrm{N}$ and $30-31^{\circ} \mathrm{W}$ )"

I. 310: Please specify that you are referring to the black dots in Fig. 9b after the statement "... along the bent-back front". In line 311 , please clarify that you are referring to the shading in Fig. 9b when discussing the vertical wind speeds. Changed following your suggestions. 
I. 322: What exactly to you mean by "on the jet stream side". Changed to "facing the jet stream core"

I. 335: Fig. 10b is a vertical cross section from south to north. So, how is it possible to see the "western edge of the cloudy area"? Changed to "southern edge of the cloudy area"

Interactive

I. 354: Can you quantify a bit how much too low? The sentence is now "...whereas the comment cloud tops are generally $1 \mathrm{~km}$ too low in NODIA."

I. 359: Is this air mass between the warm front and the Greenland plateau really dry? I agree that radar does not detect any precipitation, but I am not convinced that this airmass is dry. Also, it would be interesting to know whether this air mass (especially in the lower troposphere) is the cold conveyor belt of the cyclone. We agree that the cloud radar observation can only infer the absence of clouds. However, dropsondes show relative humidity as low as $20 \%$. The air mass is therefore quite dry. The sentence is now "These observations combined with dropsonde measurements ..."

I. 360: Please explain why the dry air mass is absent. An explanation as in I. 155 would be helpful. We added "(cutting off the diabatic cooling reduces evaporation of frozen hydrometeors under the warm front)".

I. 375: Could you explain why the ascents in the WCB outflow are solely due to cloud diabatic processes and not due to frontal dynamics. I think the statement in its current form is very strong and should be reconsidered carefully. Changed to "ascents in the WCB outflow do not occur in the absence of cloud diabatic processes."

I. 386: To support the statement that especially anticyclonic segments are associated with negative PV: Could you indicate the location of anticyclonic and cyclonic segments in Fig. 9d? At 02:00 UTC, rapid anticyclonic segments are located at around $4 \mathrm{~km}$ altitude, while rapid cyclonic segments are almost absent (Fig. 7). The former correspond well to the updrafts and to the negative PV values in Fig. 9, while the (few) latter are 
located further westward and not shown for the sake of clarity.

I. 390: Schemm et al. (2013) performed idealized moist and dry simulations of a baroclinic wave. Their results, in particular with respect to the northwestern edge of the ridge are very similar to the results of this study. Please consider to reference their work. Added

Interactive

comment

I. 401: The conditional instability is only mentioned here and in the abstract. Please describe already in the previous Section 5 where exactly the conditional instability can be found. It would be helpful to the reader if the regions of conditional instability were highlighted in the figures or if the latitude longitude coordinates of the unstable regions were provided. In the comment on Fig. $9 \mathrm{~b}$ in Sect. 5, we added "They both lie in a region of vertically homogeneous $\theta_{e}$ values, which promotes conditional instability."

I. 406: This is somewhat related to my previous comment on I. 307. Comparing the evolution of PV at the 320-K isentropic surface in Fig. 8, I have the impression that the negative PV is not simply advected. If this was the case the PV structure should be very similar in REF and NODIA due to conservation of PV in adiabatic flows (Figs. 8c, d). However, REF is characterized by more negative PV in the northwestern corner of the ridge than NODIA. So this clearly points to non-adiabatic processes. My suggestion is that the statement "these structures are then advected by the upper-level anticyclonic flow into the northwestern edge of the ridge" should be extended in the sense that also the non-conservative processes are at least mentioned. We agree. As tracking the negative PV structures would require different tools than the Lagrangian trajectories and lies beyond the scope of the study, we simply state that the strcututres are "transported by the anticyclonic flow" into the northwestern edge of the ridge.

I. 411: A reference for the statement that models "struggle to represent updrafts that do not start in the boundary layer" is needed. We now refer to the study of McTaggartCowan et al. (2020).

\section{Figures}


Fig. 1: Please label at least one isobar of the MSLP field in b) and c). Added

Fig. 5: Please indicate the position of the cyclone center with a marker. This will help WCDD the reader to follow the description in Section 4.1. Also, what is the unit of the spatial frequency? Is it simply the total number of air parcels or is it the number of air parcels per area? Please clarify. The position of the cyclone center is now shown with "L" and "spatial frequency" is now "number of air parcels".

Interactive comment

Fig. 7: What exactly do mean by "number of rapid segments lies above the average"? Does it mean that it is only shown when more than 50 . Change to "above their time average".

Fig. 9: "Updrafts and potential vorticity" is a bit confusing since also other parameters are shown. Please consider to remove or replace the first sentence of the caption. Changed to "Results"

\section{References}

Browning, K. A., M. E. Hardman, T. W. Harrold, and C. W. Pardoe, 1973: The structure of rainbands within a mid-latitude depression. Quarterly Journal of the Royal Meteorological Society, 99 (420), 215-231, doi:10.1002/qj.49709942002.

Browning, K. A., 1986: Conceptual Models of Precipitation Systems. Wea. Forecasting, 1, 23-41, https://doi.org/10.1175/1520-0434(1986)001<0023:CMOPS>2.0.CO;2.

Eckhardt, S., A. Stohl, H. Wernli, P. James, C. Forster, and N. Spichtinger, 2004: A 15-Year Climatology of Warm Conveyor Belts. J. Climate, 17, 218237,https://doi.org/10.1175/1520-0442(2004)017<0218:AYCOWC>2.0.CO;2.

Harrold, T. W., 1973: Mechanisms influencing the distribution of precipitation within baroclinic disturbances. Quarterly Journal of the Royal Meteorological Society, 99(420), 232-251, doi: 10.1002/qj.49709942003

McTaggart-Cowan, R., Vaillancourt, P. A., Separovic, L., Corvec, S., and Zadra, A., 
2020: A Convection Parameterization for Low-CAPE Environments, Mon. Weather Rev., in press, https://doi.org/10.1175/MWR-D-20-0020.1

WCDD

Schäfler, A., Craig, G., Wernli, H., Arbogast, P., Doyle, J. D., McTaggart-Cowan, R., Methven, J., Rivière, G., Ament, F., Boettcher, M.,Bramberger, M., Cazenave, Q., Cotton, R., Crewell, S., Delanoë, J., Dörnbrack, A., Ehrlich, A., Ewald, F., Fix, A., Grams, C. M., Gray,S. L., Grob, H., Groß, S., Hagen, M., Harvey, B., Hirsch, L., Jacob, M., Kölling, T., Konow, H., Lemmerz, C., Lux, O., Magnusson, L.,Mayer, B., Mech, M., Moore, R., Pelon, J., Quinting, J., Rahm, S., Rapp, M., Rautenhaus, M., Reitebuch, O., Reynolds, C. A., Sodemann,465H., Spengler, T., Vaughan, G., Wendisch, M., Wirth, M., Witschas, B., Wolf, K., and Zinner, T.: The North Atlantic Waveguide andDownstream Impact Experiment, Bull. Amer. Meteo. Soc., 99, 1607-1637, https://doi.org/10.1175/BAMS-D17-0003.1, 2018

Schemm, S., H. Wernli, and L. Papritz, 2013: Warm Conveyor Belts in Idealized Moist Baroclinic Wave Simulations. J. Atmos. Sci., 70, 627-652, https://doi.org/10.1175/JASD-12-0147.1.

Interactive comment on Weather Clim. Dynam. Discuss., https://doi.org/10.5194/wcd-2020-53, 2020. 